\section{Aneurysma spurium der Aorta ascendens mit sekundärer Perforation in den rechten Ventrikel}

Ein Aneurysma spurium der Aorta ascendens ist eine seltene, aber gefährliche Komplikation nach einem herzchirurgischen Eingriff. Die Leckage der Aorta kann an verschiedenen Stellen auftreten, zum Beispiel an der Aortotomie für einen Aortenklappenersatz, an der Insertionsstelle eines aortokoronaren Bypasses, an der Kanülierung für die Herz-LungenMaschine oder an der Nadelpunktionsstelle für die intraoperative Druckmessung (Sullivan KL et al., Chest 1988; 93: 138 -143).

Klinisch verursacht das Aneurysma spurium häufig eine pulsierende Raumforderung im Jugulum, ein retrosternales Druckgefühl, Thoraxschmerz, Myokardischämie durch Bypasskompression, Dysphagie oder Stridor. Manchmal ist das Beschwerdebild aber auch unspezifisch.

\section{Fallbericht}

Ein 49-jähriger Patient wurde mit Luftnot und diffusen Bauchschmerzen ins Krankenhaus eingewiesen. Vor 6 Monaten war ihm eine künstliche Aortenklappe eingesetzt worden.

Die Thorax-Übersichtsaufnahme im Liegen wies mit einer glattbegrenzten großbogigen Verschattung oberhalb des rechten Herzrandes auf eine Raumforderung im Mediastinum hin (Abb. 1). In der Echokardiographie konnte eine global und regional normale linksventrikuläre Pumpfunktion festgestellt werden. Die SJM-Aortenklappen-Prothese stellte sich regelrecht dar. Es fiel jedoch eine ca. $7 \mathrm{~cm}$ große Flüssigkeitsansammlung mit pulsierender Strömung vor der Aorta ascendens und dem rechten Herzen auf. Zusätzlich lag ein ca $1,5 \mathrm{~cm}$ breiter Pericarderguss vor. Diese Befunde sprachen für ein Aneurysma spurium der Aorta ascendens. Der dopplersonographische Befund über der Blutaustrittsstelle war jedoch atypisch. Es wurde ein unidirektionaler Fluss in das Aneurysma spurium nachgewiesen - der typische diastolische Rückfluss fehlte.

Zur weiteren Abklärung wurde eine Spiral-CT-Untersuchung durchgeführt

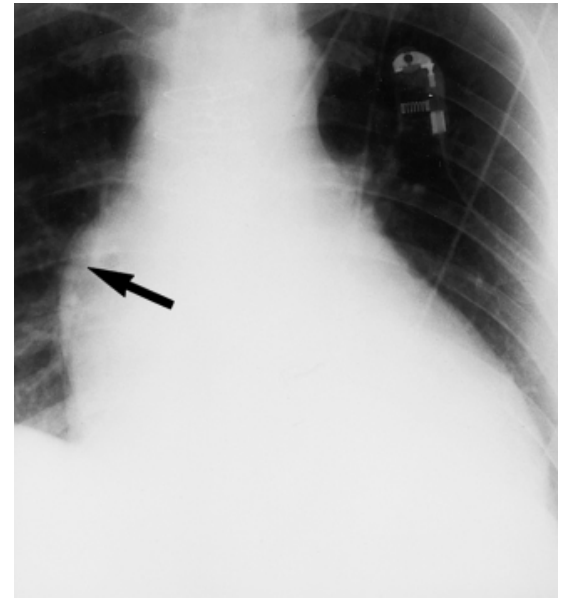

Abb. 1 Das Aneurysma spurium ruft eine bogige Verbreiterung der Mediastinalsilhouette nach rechts hervor (Pfeil).

(Schichtdicke $3 \mathrm{~mm}$, Tischvorschub $5 \mathrm{~mm}$, Rekonstruktionsinkrement $2 \mathrm{~mm}$, $120 \mathrm{ml}$ i.v.-Kontrastmittel mit $300 \mathrm{mg}$ Jod/ml, Injektionsgeschwindigkeit $3 \mathrm{ml} /$ s, Injektionsverzögerung $25 \mathrm{~s}$ ).

Das CT zeigte den bekannten $1,5 \mathrm{~cm}$ breiten Perikarderguss (Dichte 5465HE). Ein makroskopisches Leck in der Aorta ascendens war nicht erkennbar. Das retrosternale Aneurysma spurium stellte sich stark kontrastiert dar und war cirka $8 \times 8 \times 8 \mathrm{~cm}$ groß. Es hatte einen bis $\mathrm{zu} 1,5 \mathrm{~cm}$ breiten Thrombussaum (Abb. 2).

Der rechte Ventrikel wurde durch das Aneurysma spurium leicht komprimiert. Im Ausstromtrakt des rechten Ventrikels bestand eine etwa $5 \times 5 \mathrm{~mm}$ große Öffnung zwischen dem Aneurysma spurium und der rechten Kammer (Abb.3). Der dopplersonographisch festgestellte monophasische Fluss über dem Leck in der Aorta ascendens war durch diese Fistelöffnung erklärt.

Zusammenfassend wurde also mit Echokardiographie und Spiral-CT ein raumforderndes Aneurysma spurium der Aorta ascendens diagnostiziert, das durch Arrosion des rechten Ventrikels einen Anschluss an den kleinen Kreislauf entwickelt hat. Es lag ein hämorrhagischer Perikarderguss vor. Zeichen einer hämodynamischen Relevanz des Perikardergusses (erweiterte Hohlvenen, Hepatomegalie, Aszites) existierten nicht.

Als Ursache für die plötzlich eingetretene Symptomatik muss der durch die Perforation des Aneurysma spurium akut entstandene Links-Rechts-Shunt angenommen werden.

Der Patient wurde ohne weitere bildgebende Diagnostik operiert. Die Verbindung des Aneurysma spurium zum rechten Ventrikel bestätigte sich in Form einer Arrosion, die bis nahe an die Pulmonalklappe reichte. Der rechtsventrikuläre Ausflusstrakt wurde mittels Gefäßprothese rekonstruiert, der defekte Bereich der Aorta ascendens (kleines Loch an der ehemaligen lateralen Aortotomie) wurde durch eine keilförmige Gefäßprothese ersetzt.

Der operative und postoperative Verlauf war komplikationslos, der Patient wurde am zweiten postoperativen Tag auf die Normalstation verlegt und mobilisiert. Am 13. postoperativen Tag wurde er in gutem Allgemeinzustand entlassen.

\section{Diskussion}

Ein Pseudoaneurysma der Aorta ascendens nach herzchirurgischem Eingriff wurde von Sullivan et al. siebenmal in den Jahren 1963 bis 1987 beobachtet. Im gleichen Zeitraum wurden 24 Fälle in der englischsprachigen Literaur veröffentlicht.

Seitdem wurden nach unserem Wissen 48 weitere derartige Komplikationen publiziert.

In einzelnen Fällen hatte sich das unter arteriellem Druck stehende Aneurysma spurium eine Fistelverbindung zu anderen Hohlorganen bzw. durch die Haut geschaffen. Es wurde von zwei aortobronchialen Fisteln, zwei aortoösophagealen Fisteln (Razzonk A et al., Am Surg 1993, 59:818-823), von zwei arteriellen Blutungen aus der Sternalnarbe (Bridgewater BJ et al. Thorac Cardiovasc Surg 1992, 40: 45-47), von einer Fistel zum rechten Vorhof (Aoyagi $S$ et al., Thorac Cardiovasc Surg 1994, 42:58-60) und von einer Fistel zum rechten Ventrikel (Roy D et al., Tex Heart Inst J 1999; 26:140-142) berichtet. 


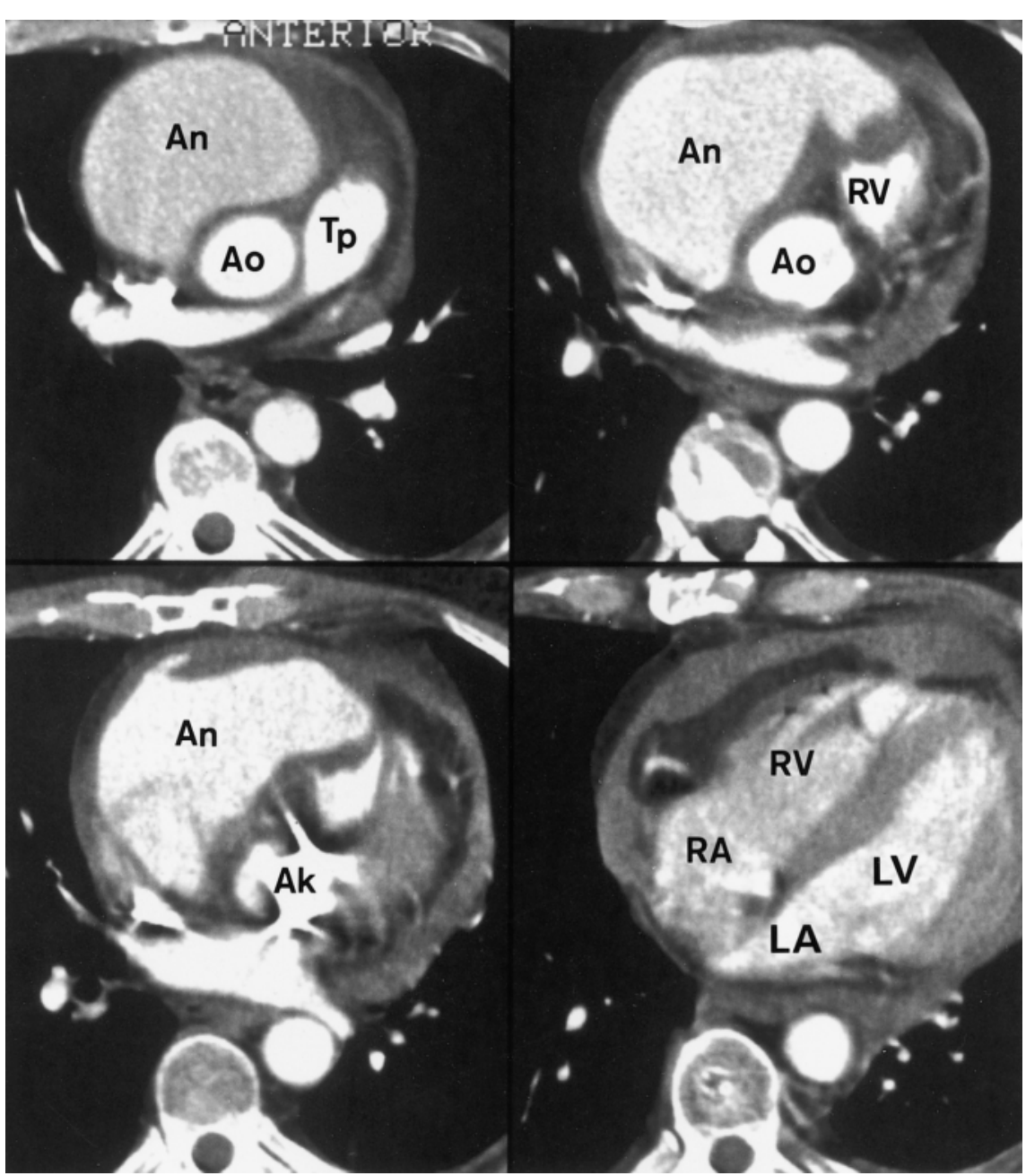

Abb. 2 Großes homogen kontrastiertes Aneurysma spurium zwischen Aorta ascendens und Sternum mit engem Kontakt zum rechten Herzen. Zirkulärer Perikarderguss. An = Aneurysma spurium, $A o=$ Aorta ascendens, $T p=$ Truncus pulmonalis, $R V=$ rechter Ventrikel, $R A=$ rechter Vorhof, LV = linker Ventrikel, LA = linker Vorhof, Ak = Aortenklappenprothese.

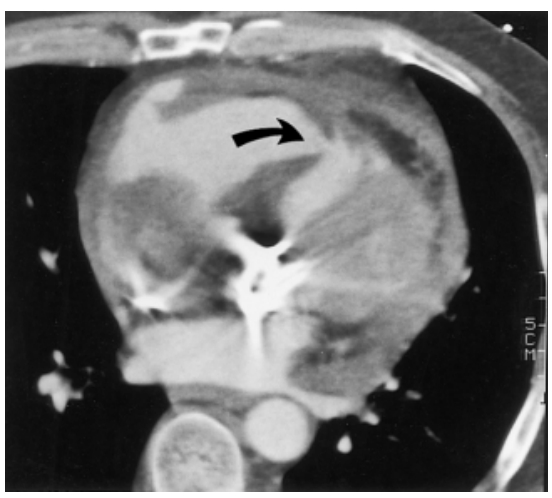

Abb. 3 Nachweis der direkten Verbindung vom Aneurysma spurium zum Ausflusstrakt des rechten Ventrikels (Pfeil).
Die klinischen Zeichen sind häufig unspezifisch. In der Thorax-Übersichtsaufnahme tritt bei einem größeren Aneurysma eine Mediastinalverbreiterung auf.

Die transthorakale Echokardiographie zeigt die pathologische mediastinale Flüssigkeitsansammlung und einen evtl. begleitenden Perikarderguss Durch die farbkodierte Duplexsonographie wird das Aneurysma spurium diagnostiziert. Duplexsonographisch lässt sich über einem Aneurysma spurium normalerweise ein biphasisches Flusssignal ableiten (systolischer Einstrom, diastolischer Rückstrom in das originäre Gefäß). Fehlt der diastolische Rückstrom, kann auf einen Abstrom des Blutes über eine Fistelverbindung geschlossen werden.

Ein Spiral-CT ist zur Diagnosesicherung und zur Operationsplanung essentiell. Es zeigt die Beziehung des Aneurysma spurium zu den umliegenden mediastinalen Strukturen. Darüber hinaus können weitere für die Operation bedeutsame Befunde, zum Beispiel Sternumosteomyelitis, Mediastinalabszess oder Verwachsungen der Aorta ascendens mit dem Sternum, durch CT dargestellt bzw. ausgeschlossen werden.

Eine MRT-Untersuchung kann bei Verwendung geeigneter Sequenzen ebenfalls sehr gut zum Nachweis eines paravalvulären Lecks herangezogen werden. Nachteilig sind lediglich die schlechtere Verfügbarkeit und die meist längeren Transportwege.

Die mit höherer Invasivität und größerem Aufwand belastete Aortographie ist nicht mehr erforderlich.

\section{A. Baier, M. Zähringer, K. Lackner, Köln} (Chest 1988, 93: 138-143). Als Infektquelle wurde eine von einer Mediastinitis ausgehende Ausbreitung per continuitatem oder eine hämatogene Besiedlung des Nahtmaterials diskutiert.

Auch bei dem hier geschilderten Patienten hatte sich zwei Monate postoperativ ein entzündlich fieberhaftes Krankheitsbild mit Begleitarthritis entwickelt, das auf Antibiotika gut ansprach. 